\title{
Modelado de las isotermas de desorción y calor isosterico de sorción en granos de café pergamino húmedo (Coffee arabica L.)
}

\author{
Modeling of desorption isotherms and isosteric heat of sorption in wet \\ parchment coffee beans (Coffee arabica L.)
}

\begin{abstract}
Ana María Ordoñez Silva
Estudiante de ingeniería agrícola, Universidad Surcolombiana. Neiva-Huila-Colombia. anita-0326@hotmail.com

Alexis Soler Campos Cerquera

Estudiante de ingeniería agrícola, Universidad Surcolombiana. Neiva-Huila-Colombia. alexis-soler@hotmail.com

Andres Gentil Collazos Escobar

Ingeniero agrícola, auxiliar de investigación del Centro Surcolombiano de Investigación en Café CESURCAFÉ. Universidad Surcolombiana. Neiva-Huila-Colombia. gentilcollazosescobar09@gmail.com

Nelson Guitierrez Guzman

Ph.D Tecnología de alimentos, docente planta Universidad Surcolombiana director del Centro Surcolombiano de Investigación en Café CESURCAFÉ.

Universidad Surcolombiana. Neiva - Huila - Colombia. ngutierrezguzman@usco.edu.co
\end{abstract}

\section{Resumen}

El secado es una de las operaciones imprescindibles para garantizar la estabilidad y calidad de los granos de café pergamino. El objetivo del presente trabajo fue modelizar las isotermas de desorción de los granos de café pergamino húmedo mediante un prototipo de secado convectivo a escala de laboratorio y la estimación del requerimiento de energía del proceso. Los datos experimentales fueron modelizados con tres expresiones empíricas que vinculan la dependencia del contenido de humedad de equilibrio y la actividad de agua con la temperatura. El calor isosterico neto de sorción fue estimado mediante la ecuación de Clausius-Clapeyron. El modelo de Iglesias y Chirife logro representar satisfactoriamente el efecto de la temperatura en las isotermas de los granos de café pergamino húmedo; presento un coeficiente de determinación ajustado mayor a 0.97 y un error cuadrático medio menor a $0.1 \mathrm{~kg} \mathrm{~kg}^{-1}$, base seca, indicando buen ajuste para fines prácticos. El calor isosterico neto de sorción disminuyo con incrementos del contenido de humedad indicando el gasto energético neto del proceso de secado.

Palavras-chave: actividad de agua; humedad de equilibrio; propiedades de sorción 


\begin{abstract}
Drying is one of the essential operations to guarantee the stability and quality of parchment coffee beans. The objective of the present work was to model the desorption isotherms of the wet parchment coffee beans by means of a prototype of convective drying at laboratory scale and the estimation of the energy requirement of the process. The experimental data were modeled with three empirical expressions that link the dependence of equilibrium moisture content and water activity with temperature. The net isosteric heat of sorption was estimated by the Clausius-Clapeyron equation. The Iglesias and Chirife model satisfactorily modeled representing the effect of temperature on the isotherms of the wet parchment coffee beans; presented a determination coefficient higher than 0.97 and a root mean square error lower than $0.1 \mathrm{~kg}$ dry basis, all of which indicate a good fit for practical purposes. The net isothermal heat of sorption decreased with increases in moisture content indicating the net energy expenditure of the drying process.
\end{abstract}

Keywords: water activity; equilibrium moisture content; sorption properties

\section{Introducción}

El café es un cultivo importante que provee una economía sostenible a los agricultores (Poltronieri \& Rossi, 2016), es considerado uno de los productos más importantes en el mundo debido a los ingresos de industrialización y exportación (Corrêa, et al., 2014). El café recién cosechado evidencia alto contenido de humedad; alrededor de $60 \%$ (base húmeda) (Resende, et al., 2009), por lo tanto, se hace imprescindible el proceso de secado para prevenir el ataque de microorganismos y fermentación que comprometería la calidad de los granos (Alves, et al., 2012).

Generalmente el secado se realiza exponiendo los granos a los rayos del sol y se debe alcanzar un contenido de humedad entre el 10 y $12 \%$ (Patiño-Velasco, et al., 2016), para garantizar la estabilidad del producto por un largo periodo de tiempo (Ghosh \& Venkatachalapathy, 2015). Sin embargo, el secado solar está condicionado por el estado del tiempo: lluvia inesperada y otros efectos de intemperie, invasión de insectos, pájaros y roedores, las cuales tienen gran influencia en la perdida y el deterioro del alimento durante la deshidratación (Ekechukwu \& Norton, 1999), tomando importancia los sistemas de secado convectivo que pueden apoyar y/o complementar el secado solar en situaciones adversas. Es una de las tecnologías conservadoras más importantes y utilizadas en la industria alimentaria (Mireles-Arriaga, et al., 2016); un aspecto negativo es la gran cantidad de energía que consume (Váquiro, 2009). Por lo tanto, la estimación del requerimiento energético del proceso se hace necesario.

Las curvas de equilibrio higroscópico son útiles para definir los límites de deshidratación del producto, estimar las alteraciones del contenido de humedad en condiciones ambientales y para predecir las condiciones óptimas de almacenamiento (Corrêa, et al., 2015), además, mediante las isotermas de sorción, el requerimiento neto de energía del proceso de deshidratación puede ser determinado (Noshad, et al., 2012).

El presente trabajo tuvo como objetivo determinar y modelizar las isotermas de sorción de los granos de café pergamino húmedo y la estimación del calor isosterico neto de sorción mediante un prototipo de secado convectivo a escala de laboratorio a temperaturas experimentales de 40 y $50{ }^{\circ} \mathrm{C}$.

\section{Materiales y métodos}

\subsection{Muestras de café}

Las muestras de café pergamino húmedo (Coffee Arabica L.) de variedad Castillo, fueron obtenidas directamente de productores del municipio del Pital, 
ubicado al sur de la región Huila a 1,538 metros sobre el nivel del mar.

\subsection{Determinación experimental de las isotermas de sorción}

CORTE VISTA PERFII

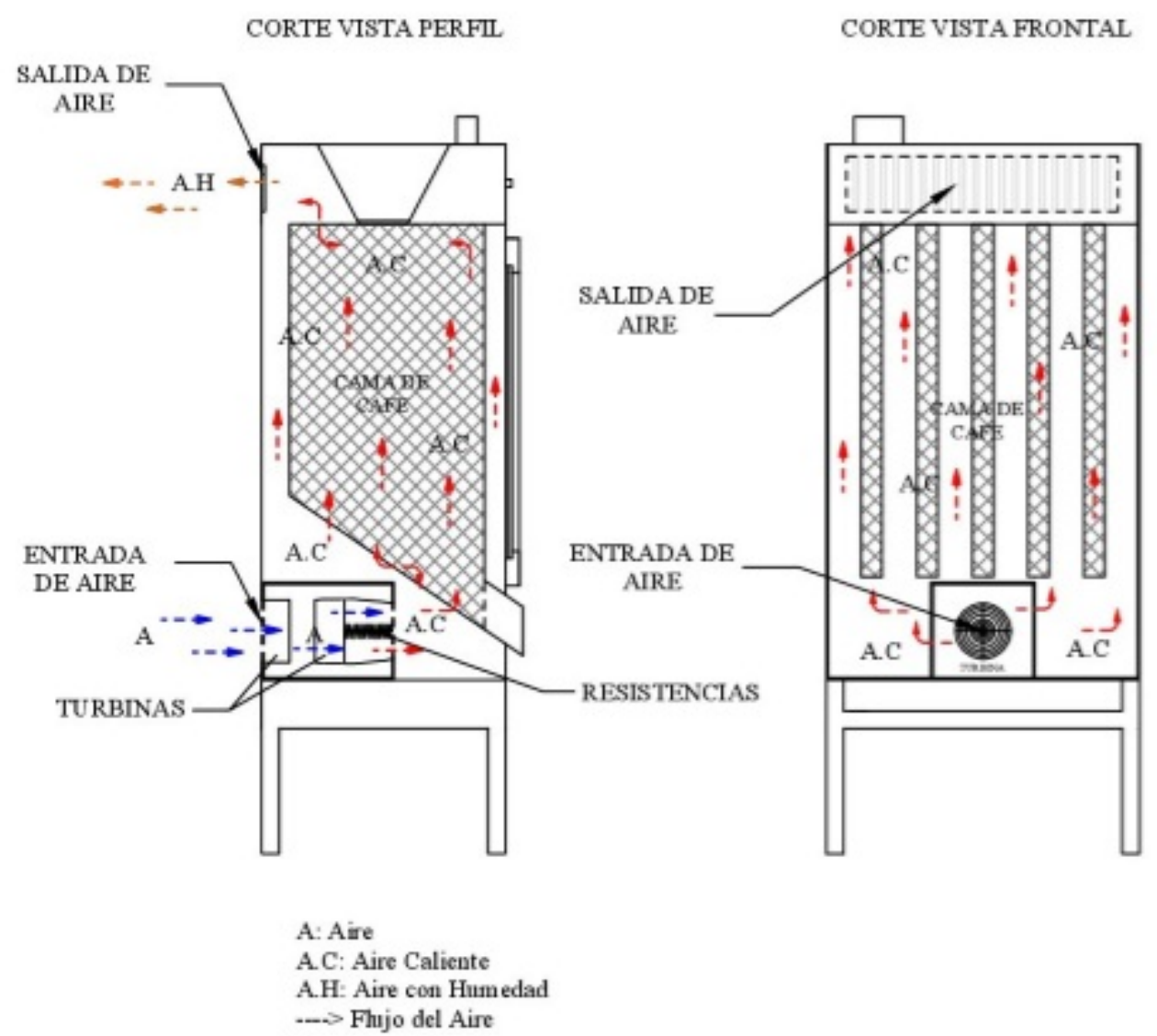

Muestras de 10.5 kilogramos $(\mathrm{kg})$ fueron deshidratadas a 40 y $50{ }^{\circ} \mathrm{C}$, haciendo uso de un prototipo de secado mecánico a escala de laboratorio, como se observa en la siguiente figura.

Figura 1. Sistema para secado mecánico de café

En la figura 1, se observa la aducción de aire ambiente al sistema de secado mediante dos turbinas, además el equipo permite control preciso del flujo y de la temperatura del aire de secado. El aire es calentado por una resistencia y la cámara de secado está compuesta por cinco celdas de 3 $\mathrm{cm}$ (centímetros) de espesor de sección cuadrada ubicadas sobre un plenum de $44 \mathrm{~cm}$ de ancho y $77 \mathrm{~cm}$ de alto; para flujo de aire uniforme. La velocidad del aire de secado fue monitoreada usando un termo anemómetro EXTECH-FieldMaster, para todos los casos la velocidad fue constante $\left(3 \mathrm{~m} \mathrm{~s}^{-1}\right)$.

Las muestras de café pergamino húmedo fueron obtenidas durante el proceso de secado; empacadas en bolsas plásticas herméticas y dispuestas en jarros de vidrio durante 48 horas a temperatura ambiente $\left(\sim 25{ }^{\circ} \mathrm{C}\right)$ antes de tomar mediciones de actividad de agua (Bon, et al., 2012); para garantizar la redistribución de la humedad de la muestra (Zhang, et al., 2015).

\subsubsection{Medición de contenido de humedad}

La medición del contenido de humedad de las muestras se realizó por triplicado haciendo uso del determinador de humedad kett PM 450.

\subsubsection{Medición de actividad de agua}

La medición de actividad de agua puntual se 
realizó por triplicado haciendo uso del Vapor Sorption Analizer (VSA) Aqualab Decagon; previo a la medición, el sensor de punto de roció se verifico empleando cuatro soluciones acuosas saturadas 13.41 molal LiCl $\left(0.250 \pm 0.003 \mathrm{a}_{w}\right), 8.57 \mathrm{~m} \mathrm{LiCl}(0.50 \pm$ $\left.0.003 \mathrm{a}_{w}\right), 6.0 \mathrm{~m} \mathrm{NaCl}\left(0.760 \pm 0.003 \mathrm{a}_{w}\right)$ y $2.33 \mathrm{~m}$ $\mathrm{NaCl}\left(0.92 \pm 0.003 \mathrm{a}_{w}\right)$.

Aplicando esta metodología, catorce puntos experimentales (actividad de agua/contenido de humedad) se obtuvieron para cada temperatura (40 ${ }^{\circ} \mathrm{C}$ y $\left.50{ }^{\circ} \mathrm{C}\right)$. Los datos variaron de 0.126 a $1.175 \mathrm{~kg}$ $\mathrm{kg}^{-1}$ (base seca) y 0.554 a 0.997 para actividades de agua.

\subsection{Modelado de las isotermas de desorción}

Los datos experimentales fueron modelados con tres expresiones matemáticas para describir la desorción de los granos de café pergamino húmedo. Los modelos matemáticos se presentan en la tabla 1 , donde $\mathrm{X}_{e}$ es el contenido de humedad de equilibrio $\left(\mathrm{kg} \mathrm{kg}^{-1}\right.$, b.s. $), \mathrm{a}_{i}$ es la actividad de agua $\mathrm{y} \mathrm{b}_{i}$ son los parámetros empíricos de los modelos.

Tabela 1. Expresiones matemáticas para la modelización de granos de café pergamino húmedo

\begin{tabular}{cccc}
\hline Modelo & Referencia & Expresión & Ecuación \\
\hline Iglesias y Chirife & (Caballero-Cerón, et al., 2017). & $X_{e}=b_{1}+b_{2}\left(\frac{a_{w}}{1-a_{w}}\right)$ & $(1)$ \\
\hline DLP & (Shittu, et al., 2015). & $X_{e}=b_{0}+b_{1} x+b_{2} x^{2}+b_{3} x^{3}$ & $(2)$ \\
& & $x=\ln \left(-\ln a_{w}\right)$ & \\
\hline Polynomiall & (Mousa, et al., 2012). & $X_{e}=b_{0}+b_{1} a_{w}+b_{2} a_{w}^{2}+b_{3} a_{w}^{3}$ & $(3)$ \\
\hline
\end{tabular}

${ }^{*}$ Se ha considerado que los parámetros de las ecuaciones empíricas tienen una dependencia lineal con la temperatura $\left(b_{i} * T+b_{i \cdot 1}\right)$

\subsubsection{Estimación de parámetros y análisis estadístico}

Para el ajuste de los modelos matemáticos se realizó análisis de regresión no lineal con la herramienta Curve Fitting de Matlabß R2017a (The MathWorks Inc., Natick, MA, USA), se usó para identificar los parámetros del modelo y calcular los intervalos de confianza del $95 \%$. El coeficiente de determinación ajustado $\left(\mathrm{R}_{a d j}^{2}\right)$ y la raíz del error cuadrático medio (RMSE) se utilizaron para evaluar la bondad de ajuste de los modelos. Según Bastığlu et al (2017) se considera que un modelo es aceptable si $R M S E$ se encuentra por debajo del $10 \%(0.1 \mathrm{~kg}$ $\mathrm{kg}^{-1}$, b.s.) y $\mathrm{R}^{2}$ mayor a 0.9. De manera general altos valores de $\mathrm{R}^{2}$ y $\mathrm{R}_{\text {adj }}^{2}$ y bajos valores de RMSE se considera como bondad de ajuste alta (Yang, et al., 2015).

\subsection{Calor isosterico neto de sorción}

Se realizó la estimación del calor isosterico neto de sorción mediante la ecuación de Clausius-Clapeyron.

$$
q_{s n}=-R\left[\frac{\partial\left(\ln a_{w}\right)}{\partial\left(\frac{1}{T}\right)}\right] x e
$$

Donde $\mathrm{q}_{s n}$ es el calor isosterico neto de sorción ( $\left.\mathrm{J} \mathrm{mol}{ }^{-1}\right), a_{w}$ actividad de agua, $T$ temperatura absoluta (K), $R$ constante universal de los gases $\left(8.314 \mathrm{~J} \mathrm{~mol}^{-1} \mathrm{k}^{-1}\right)$ y x contenido de humedad $(\mathrm{kg}$ $\mathrm{kg}^{-1}$ b.s.). El calor isosterico neto de sorción se determinó con la ecuación (4) utilizando la gráfica $\ln \left(a_{w}\right)$ frente a $1 / T$ y calculando la pendiente a contenido de humedad constante (Bensebia \& Allia, 2016); (Tadapaneni, et al., 2017); (Baptestini, et al., 2017); (Taoufik, et al., 2017); (Domian, et al., 2018), la pendiente resultante debe ser multiplicada por $-R$ (Bonner \& Kenney, 2013); (Moreira, et al., 2016).

\section{Resultados y discusión}

La tabla 2 presenta los tres modelos matemáticos evaluados de mejor ajuste a los datos experimentales, considerando el efecto de la temperatura; el modelo de Iglesias y Chirife podría considerarse el que mejor representa la desorción en los granos de café pergamino húmedo, debido a su alto valor de $\mathrm{R}_{a j s}^{2}$ 0.975 y RMSE $0.049 \mathrm{~kg} \mathrm{~kg}^{-1}$ b.s. Los demás modelos 
resultaron con valores $\mathrm{R}_{a j s}^{2}$ inferiores al modelo de Iglesias y Chirife a pesar de presentar residuos adecuados. Los intervalos de confianza muestran que todos los parámetros del modelo fueron estadísticamente significativos a un nivel de confianza del 95\%; lo que indica un buen ajuste para fines prácticos (Baptestini, et al., 2017).

Tabela 2. Bondad de ajuste y parámetros del modelo

\begin{tabular}{ccccc}
\hline Modelo & Parámetros & Intervalos de Confianza 95\% & $\mathbf{R}_{a d j}^{2}$ & RMSE \\
\hline Iglesias y Chirife & $b_{2.1}=0.346$ & {$[0.298,0.394]$} & & \\
& $b_{2}=-1.001 \times 10^{-3} K^{-1}$ & {$\left[-1.151 \times 10^{-3},-8.503 \times 10^{-4}\right]$} & 0.975 & 0.049 \\
& $b_{1}=3.106 \times 10^{-4} K^{-1}$ & {$\left[2.591 \times 10^{-4}, 3.622 \times 10^{-4}\right]$} & & \\
\hline & $b_{3}=0.208 K^{-4}$ & {$[0.152,0.263]$} & & \\
Polynomial & $b_{2}=-0.448 K^{-1}$ & {$[-0.577,-0.319]$} & & \\
& $b_{1}=0.318 K^{-1}$ & {$[0.221,0.416]$} & 0.887 & 0.105 \\
& $b_{0.1}=2.668$ & {$[1.158,4.177]$} & & \\
\hline DLP & $b_{0}=-0.082 K^{-1}$ & {$[-0.107,-0.057]$} & & \\
& $b_{3}=-0.018$ & {$[-0.019,-0.017]$} & 0.958 & 0.063 \\
& $b_{0}=4.599 \times 10^{-4} K^{-1}$ & {$\left[3.96 \times 10^{-4}, 5.239 \times 10^{-4}\right]$} & & \\
\hline
\end{tabular}

La figura 2 presenta las isotermas de desorción a las temperaturas experimentales $40^{\circ} \mathrm{C}$ y $50^{\circ} \mathrm{C}$; las curvas de equilibrio higroscópico resultantes son de tipo II acorde con la clasificación de Brunauer (1940), se evidencia que el efecto de la temperatura en las isotermas promueve diferencias significativas en el contenido de humedad de equilibrio, es decir con incremento de temperatura a un valor de actividad de agua constante, el contenido de humedad de equilibrio se reduce (Baptestini, et al., equilibrio.

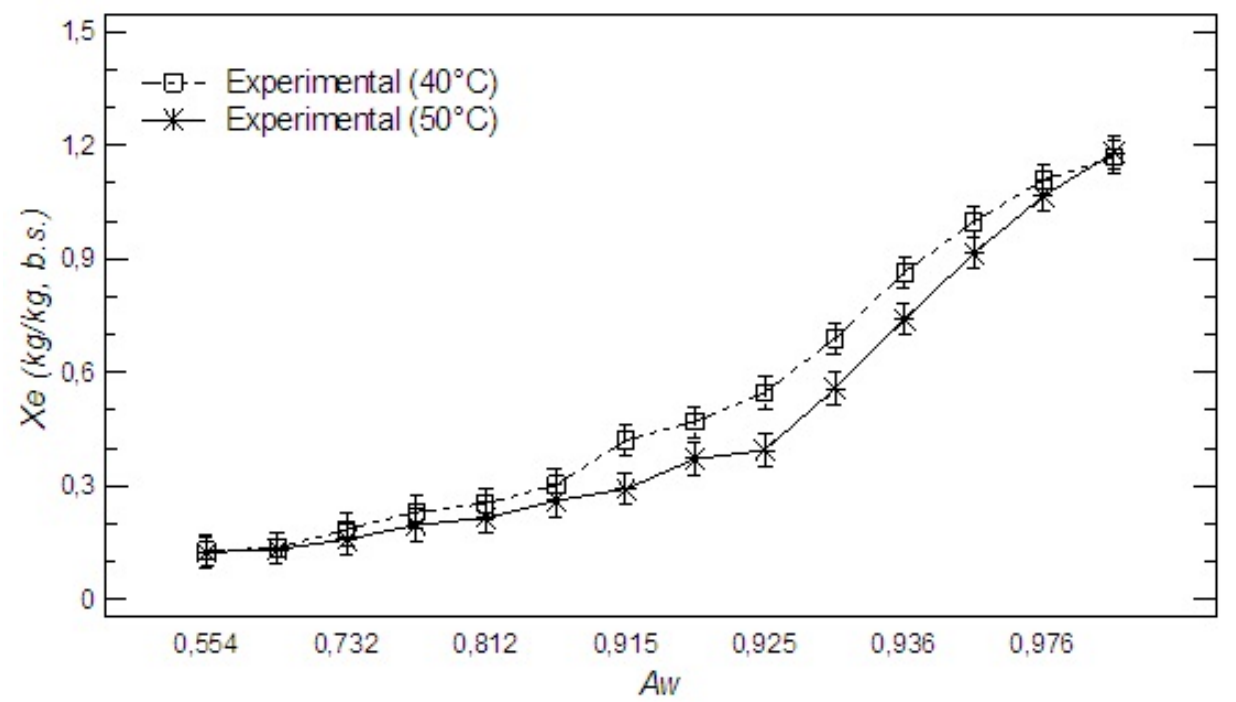

Figura 2. Isotermas de desorción experimental granos de café pergamino a temperaturas de $40^{\circ} \mathrm{C}$ y $50^{\circ} \mathrm{C}$
2017); este hecho puede ser atribuido a que la temperatura activa las moléculas de agua lo que les permite separarse de sus sitios de sorción (Corrêa, et al., 2014). Bastıŏlu et al (2017) mencionan que el comportamiento puede ser atribuido a que las fuerzas atractivas entre las moléculas disminuyeron debido al aumento en la energía cinética de las moléculas de agua a más altas temperaturas, disminuyendo así el contenido de humedad de 
En la figura 3 se observa la generalización capaz de representar satisfactoriamente el fenómeno del modelo de Iglesias y Chirife vinculando el de desorción en función de actividades de agua efecto de la temperatura en las isotermas de comprendidas entre $0.554-0.997$ y temperaturas de desorción, se evidencia que la expresión general es 40 y $50^{\circ} \mathrm{C}$.

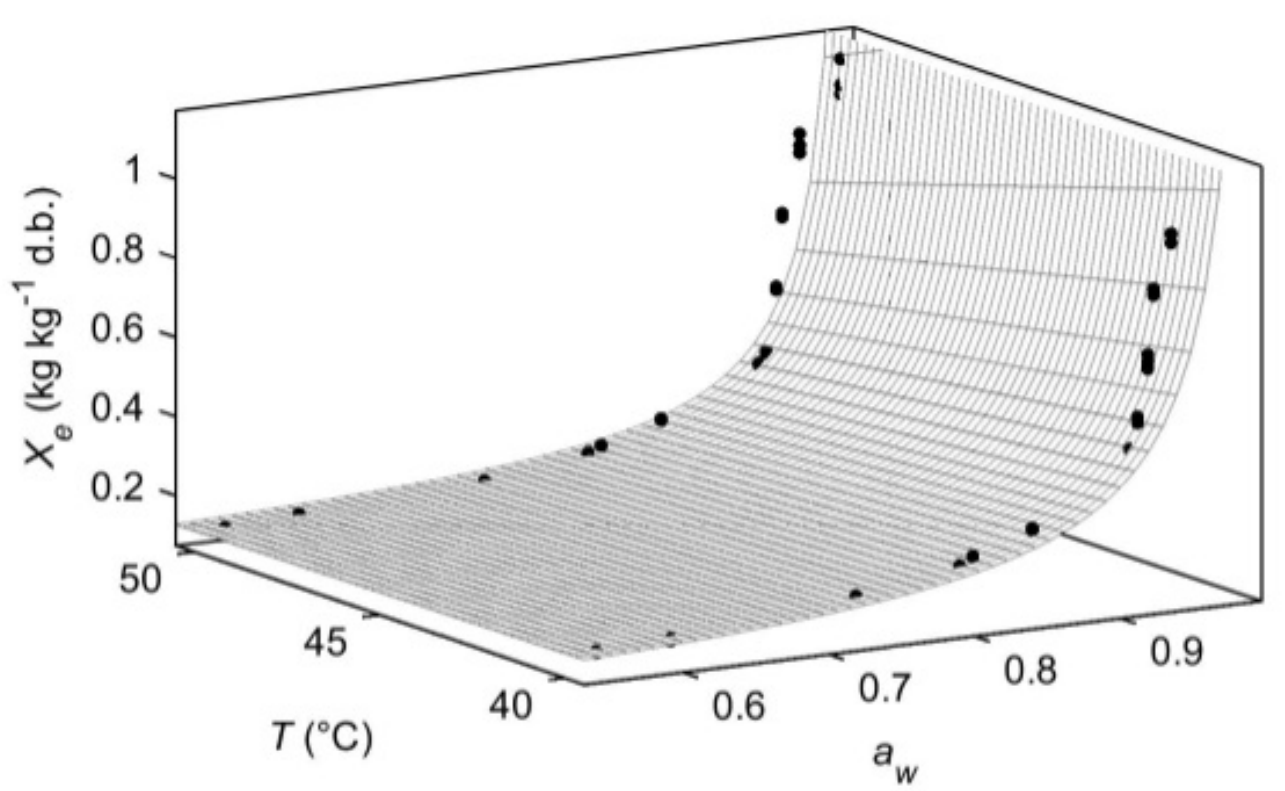

Figura 3. Isotermas de desorción experimental y estimado con el modelo Iglesias y Chirife en granos de café pergamino húmedo a temperatura de $40^{\circ} \mathrm{C}$ y $50^{\circ} \mathrm{C}$.

La figura 4 presenta la variación del calor del calor de sorción con el incremento del contenido isosterico neto de sorción en función del contenido de humedad de equilibrio. de humedad de equilibrio, se observa la disminución

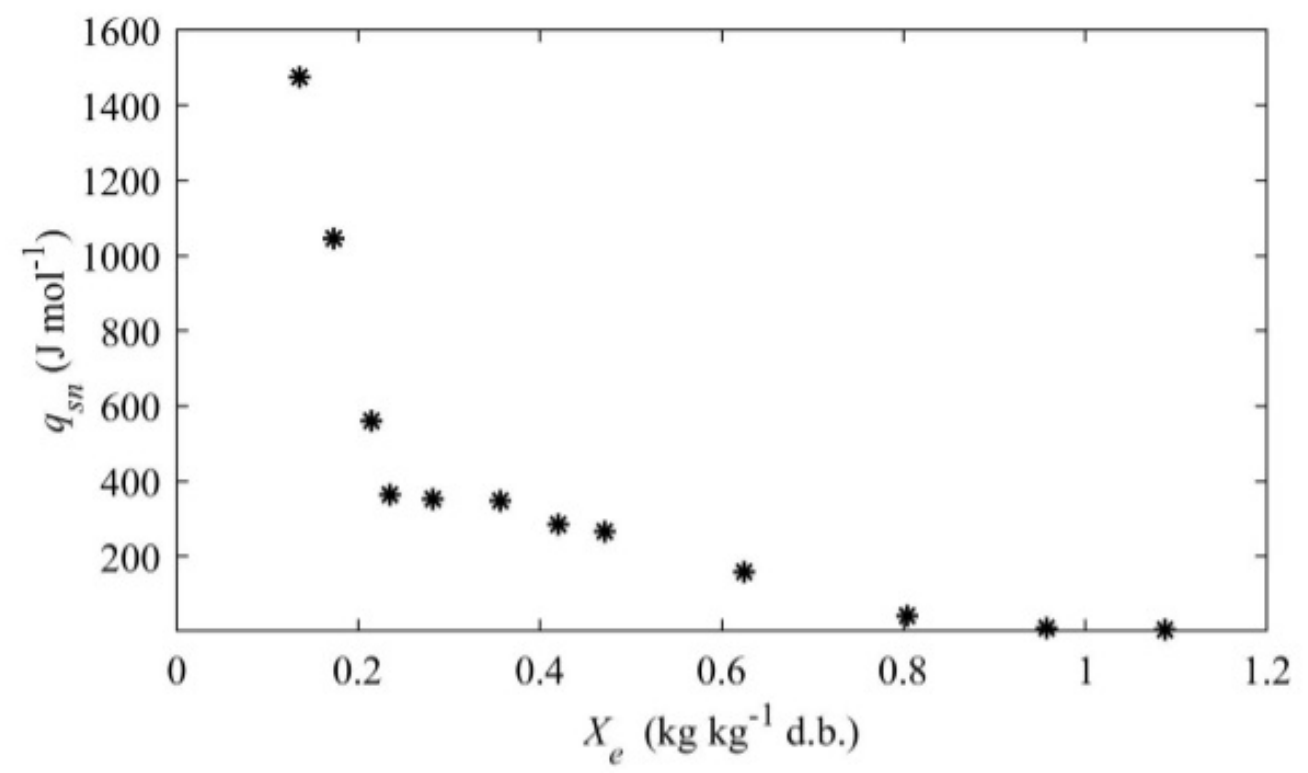

Figura 4. Variación del calor isosterico neto de sorción en granos de café pergamino húmedo 
La energía requerida para eliminar el agua del producto es mayor en niveles bajos de humedad (Goneli, et al.,2013), este hecho puede ser explicado al considerar que a bajos contenidos de humedad la sorción ocurre en los sitios disponibles más activos dando lugar a gran energía de interacción (Cano-Higuita, et al., 2015); (Villa-Vélez, et al., 2012), la superficie del producto se encuentra cubierta de moléculas de agua, formando una capa monomolecular; a medida que incrementa el contenido de humedad, las moléculas de agua se unen químicamente a los sitios activos y la sorción comienza a ocurrir en sitios menos activos, con menor energía de interacción y, en consecuencia, menor calor isostérico de sorción (Baptestini, et al 2017); (Corrêa, et al., 2010), un efecto similar al reportado por Corrêa et al (2010); Goneli et al (2013) en granos de café pergamino, Baptestini et al (2017) en café tostado molido y en diferentes productos agrícolas (Mousa, et al., 2012); (Červenka, et al., 2015); (Bon, et al., 2012); (Ferreira de Souza, et al., 2014); (Taoufik, et al., 2017).

\section{Conclusiones}

Los resultados permitieron concluir que el contenido de humedad de equilibrio disminuye con el aumento de la temperatura a valores de actividad de agua constante. Entre los modelos empíricos evaluados el modelo de Iglesias y Chirife represento de manera satisfactoria las isotermas de desorción de los granos de café pergamino, debido a la alta correspondencia y bondad de ajuste a los datos experimentales. Las isotermas se comportaron de manera típica para los granos de café pergamino. El calor isosterico neto de sorción disminuyo con incrementos del contenido de humedad, indicando el requerimiento energético del proceso de secado, un aproximado de 1,500 $\mathrm{J} \mathrm{mol}^{-} 1$ para deshidratar los granos en un rango de humedad de 1.175 a 0.126 $\mathrm{kg} \mathrm{kg}^{-} 1$ b.s. Las isotermas de sorción y el calor isosterico fueron similares a los informados para café y otros productos agrícolas.

\section{Referencias bibliográficas}

Alves, E. G., Isquierdo, P. E., Borém, M. F., Siqueira, C. V., Oliveira
Andrade, T. E., 2013. Drying kinetics of natural coffee for different temperature and low relative humidity. Coffee Science, Lavras 8(2):238-247. Consultado el 2 de agosto de 2018. https://www.researchgate.net/publication/2893277 20_Drying_kinetics_of_natural_coffee_for_diffe rent_te mperatures_and_low_relative_humidity

Baptestini, M. F., Corrêa, P. C., Horta de Oliveira, Cecon, P. R., Ferreira, S. F., 2017. Kinetic modeling of water sorption by roasted and ground coffee. Acta Scientiarum Agronomy.

Doi: 10.4025/actasciagron.v39i3.32576

Bastıoğlu, A. Z., Koç, M., Ertekin, F. G., 2017. Moisture sorption isotherm of microencapsulated extra virgin olive oil by spray drying. Food Measure. DOI 10.1007/s11694-017-9507-4

Bensebia, O., Allia, K., 2016. Analysis of adsorption-desorption moisture isotherms of rosemary leaves. Journal applied Research on Medicinal And Aromatics Plants. http://dx.doi.org/10.1016/j.jarmap.2016.01.005

Bon, J., Vaquiro, H. A., Mulet, A., 2012. Modeling sorption isotherms and isosteric heat of sorption of mango pulp cv. tommy atkins. Biotecnología en el Sector Agropecuario y Agroindustrial. Vol $10 \quad$ No. 2 (34 43). Consultado el 10 de septiembre de 2017. http://www.scielo.org.co/scielo.php?script=sci_artt ext\&pid=S1692-35612012000200005

Bonner, I. J., Kenney, K. L., 2013. Moisture sorption characteristics and modeling of energy sorghum (Sorghum bicolor (L.) Moench. Journal of Stored Products Research. http://dx.doi.org/10.1016/j.jspr.2012.11.002

Brunauer, S., Deming, S. L., Deming, E. W., Teller, E., 1940. On a Theory of the van der Waals Adsorption of Gases. J. Am. Chem. Soc.. DOI: $10.1021 /$ ja01864a025

Caballero-Cerón, C., Serment-Moreno, V., D. P., Velazquez, G., Torres, J. A. Welti-Chanes, J., 2017. 
Hygroscopic properties and glass transition of dehydrated mango, apple and banana. J Food Sci Technol. https://doi.org/10.1007/s13197-017-2963-3

Cano-Higuita, D. M., Villa-Vélez, H. A., Telis-Romero, J., Váquiro, H. A., Nicoletti, T. V. R., 2015. Influence of alternative drying aids on watersorption of spray dried mango mix powders: A thermodynamic approach. Food and Bioproducts Processing. http://dx.doi.org/10.1016/j.fbp.2013.10.005

Červenka,, L., Hloušková, L., Žabčíková, S., 2015. Moisture adsorption isotherms and thermodynamic properties of green and roasted Yerba mate (Ilex paraguariensis). Food Bioscience. https://doi.org/10.1016/j.fbio.2015.10.001

Corrêa, P. C., Reis, T. M. F., Horta de Oliveira, G. H., Rodrigues de Oliveira, A. P. L., Botelho, F. M., 2015. Moisture desorption isotherms of cucumber seeds: modeling and thermodynamic properties. Journal of Seed Science. http://dx.doi.org/10.1590/2317-1545v37n31495 49

Corrêa, P.C, Botelho, M. F., Botelho, C. Silva de C., Goneli, D. L. A., 2014. Isotermas de sorção de água de frutos de Coffea canephora. Revista Brasileira de Engenharia Agrícola e Ambiental., Campina Grande, PB, UAEA/UFCG. DOI: http://dx.doi.org/10.1590/1807-1929/agri ambi.v18n10p1047-1052

Corrêa, P.C, Goneli, L. A., Junior, A. Oliveira, H. G., Valente, D., 2010. Moisture sorption isotherms and isosteric heat of sorption of coffee in different processing levels. International Journal of Food Science and Technology. doi:10.1111/j.1365-2621.2010.02373.x

Domian, E., Brynda-Kopytowska, A., Ciesla, J., Górska, A., 2018. Effect of carbohydrate type on the DVS isotherm-induced phase transitions in spray-dried fat-filled pea protein-based powders. Journal of Food Engineering. DOI: https://doi.org/10.1016/j.jfoodeng.2017.11.012

Ekechukwu, O. V., Norton, B., 1999.
Review of solar-energy drying systems II: an overview of solar drying technology. Energy Conversion \& Management. DOI: https://doi.org/10.1016/S0196-8904(98)00093-4

Ferreira de Souza, S. J., Váquiro, H. A., Villa-Vélez, H. A., Polachini, T. C., Telis-Romero, J., 2014. Physical, Thermal and Water-Sorption Properties of Passion Fruit Seeds. International Journal of Food Engineering. doi 10.1515/ijfe-2014-0138

Ghosh, P., Venkatachalapathy, N., 2015. Changes in physico-chemical properties of coffee due to hot air assisted microwave drying. International Journal of Processing and Post-Harvest Technology. DOI: 10.15740/HAS/IJPPHT/6.1/69-79

Goneli, A.L.D., Corrêa, P. C., Oliveira, G.H.H., Afonso Júnior, P.C., 2013. Water sorption properties of coffee fruits, pulped and green coffee. LWT - Food Science and Technology. DOI: http://dx.doi.org/10.1016/j.lwt.2012.09.006

Mireles-Arriaga, A. I., Ruiz-López, I. I., Hernández-García, P. A., Espinosa-Ayala, E., López-Martínez, L. X., Márquez-Molina, O., (2016). The impact of convective drying on the color, phenolic content and antioxidant capacity of noni (Morinda citrifolia L.). Food Science and Technology. DOI: http://dx.doi.org/10.1590/1678-457X.00415

Mousa, W., Mohamad, F., Jinap, S. G., Mohd, H., Radu, S., 2012. Sorption isotherms and isosteric heats of sorption of Malaysian paddy. J Food Sci Technol DOI 10.1007/s13197-012-0799-4

Noshad, M., Shahidi, F., Mohebbi, M., Mortazavi, S. A., 2012. Desorption isotherms and thermodynamic properties of fresh and osmotic-ultrasonic dehydrated quinces. Journal of Food Processing and Preservation ISSN 1745-4549. doi:10.1111/j.1745-4549.2011.00671.x

Patiño-Velasco, M. M., Pencue-Fierro, E. L., Vargas-Cañas, R., 2016. Determinación del contenido de humedad en granos de café pergamino 
seco utilizando speckle dinámico. Biotecnología en el Sector Agropecuario y Agroindustrial. DOI:10.18684/BSAA(14)84-91

Poltronieri, P., Rossi, F., 2016. Challenges in Specialty Coffee Processing and Quality Assurance. Journal Challenges. DOI: 10.3390/challe7020019

Resende, O., Arcanjo, V. R., Siqueira, C. V., Rodrigues, S., 2009. Modelagem matemática para a secagem de clones de café (Coffea canephora Pierre) em terreiro de concreto. Acta Scientiarum. Agronomy. DOI: 10.4025/actasciagron.v31i2.588

Shittu, T. A., Idowu-Adebayo, F., Adedokun, I. I., Alade, O., $2015 . \quad$ Water vapor adsorption characteristics of starch-albumen powder and rheological behavior of its paste. Nigerian Food Journal. DOI: http://dx.doi.org/10.1016/j.nifoj.2015.04.014

Tadapaneni, R. K., Yang, R., Carter, B., Tang, J., 2017. A new method to determine the water activity and the net isosteric heats of sorption for low moisture foods at elevated temperatures. Food Research International. http://dx.doi.org/10.1016/j.foodres.2017.09.070

Taoufik, F., Hadek, M., Hnini, M. C., Benchanaa, M'B., Hammioui, M., Hassani, I. L. M., 2017. Sorption isotherms and isosteric heats of sorption of mint variety (Mentha viridis) leaves and stems: Experimental and mathematical investigations. The European physical journal special topics. DOI: 10.1140/epjst/e2016-60180-1

Váquiro, H A., 2009. Contribución al estudio y optimización del secado intermitente: aplicación al secado de mango (Mangifera indica L var. Tommy Atkins). Thesis for: Doctor, Universidad Politécnica de Valencia, Departamento de Tecnología de Alimentos. Valencia, España Advisor: José Bon Corbín, Antonio Mulet Pons. DOI: $10.4995 /$ Thesis/10251/6062

Villa-Velez, H. A., Váquiro, H. A., Bon, J., Telis-Romero, J., $2012 . \quad$ Modelling thermodynamic properties of Banana waste by analytical derivation of desorption isotherms. International Journal of Food Engineering. DOI: https://doi.org/10.1515/1556-3758.2191

Yang, Z., Zhu, E., Zhu, Z., 2015. Water desorption isotherm and drying characteristics of green soybean. Journal of Stored Products Research. https://doi.org/10.1016/j.jspr.2014.10.006

Zhang, L., Da-Wen, S., Zhang, Z., 2015. Methods for Measuring Water Activity (aw) of Foods and Its Applications to Moisture Sorption Isotherm Studies. Critical Reviews in Food Science and Nutrition. DOI: http://dx.doi.org/10.1080/10408398.2015.1108282

La Revista Ingeniería y Región cuenta con la Licencia Creative Commons Atribución (BY), No Comercial (NC) y Compartir Igual (SA)

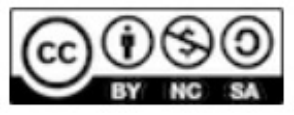

